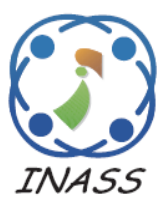

\title{
Base Station Positioning in Wireless Sensor Network to aid Cluster Head Selection Process
}

\author{
Achyut Shankar $^{1 *} \quad$ Jaisankar Natarajan ${ }^{1}$ \\ ${ }^{1}$ School of Computing Science and Engineering, Vellore Institute of Technology University, Vellore, India \\ * Corresponding author’s Email: achyutshankar@gmail.com
}

\begin{abstract}
In this paper, we propose an (SAPSO) Self-Adaptive Particle Swarm Optimization algorithm to solve the base station positioning problem. This algorithm is used to minimize the distance between the base station and cluster head owing to maximizing the energy. Moreover, the proposed algorithm is compared with many other conventional algorithms such as Artificial Bee Colony-Dynamic Scout bee (ABC-DS) algorithm, Genetic algorithm (GA), Particle swarm optimization (PSO) and Firefly with Dual Update Process (FFDUP) algorithm. Also, empowering SAPSO helps to find the optimal location of the base station to conserve energy in a noteworthy manner to maximize the network lifetime and prolong network connectivity regarding minimizing distance, delay and maximizing the security, energy. Our simulation results demonstrate that the proposed SAPSO algorithm performs better than the other conventional algorithm.
\end{abstract}

Keywords: SAPSO, WSN, Clustering, Cluster head, Base station.

\section{Introduction}

Wireless Sensor Network (WSN) is most commonly used in various applications such as military, industrial, health monitoring and vehicular tracking [1,2]. Moreover, WSN consists of thousand sensors with small nodes, and it has the advantages like low cost, high storage capacity, sensing and consumes low power while comparing with other computer networks [3]. Generally, the main aim of WSN is to gather the data from the environments and transmit the data to the site for analyzing the data [46]. Here, the sensor node is used to measure the environmental condition and to transmit the gathered data to the node is called base station [7-9].

To overcome this drawback few sensor networks utilize the base stations to provide inexhaustible power supply [10-12]. Generally, a base station is used to gather the data from the sensors to communicate with the central authority [13,14]. The base station act as static, but in some cases, it acts as mobile to gather the data from sensor nodes [15-17]. Here, the positioning of the base station has an impact on the lifetime of the sensor to transmit data for decision-making process [18,19]. Besides, the distance between the sensor nodes and the base station is decreased to minimize the energy transmission [20-22]. The minimum energy and maximum lifetime of networks can be achieved by the optimal location of a base station. Moreover, the nodes consume lesser energy while nearer to the base station [23,24]. (Worldwide Interoperability for Microwave Access) WiMAX has intended to present the maximum speed broadband wireless admittance used for the permanent base stations and the mobile stations [25]. Furthermore, the positioning of the base station can affect the network performance such as delay, throughput and energy consumption [26-28].

In addition, clustering is one of the essential techniques to save energy consumption $[14,29,30]$. Generally, a cluster head is represented as a controller of nodes in the cluster group. Meanwhile, clustering technique is used to reduce the network load and hence the nodes preserve a significant amount of energy [31,32]. In general, sensor nodes belong to one cluster, and sensor nodes send data to the cluster head, then the cluster head forwards the data to the 
base station based on multi-hop communication [33,34].

In this paper, we have proposed SAPSO algorithm to determine the optimal position of the base stations. The optimal position of the base station has been identified in reference to maximizing the lifetime of a sensor network. Moreover, the selection of the cluster head also plays an important role for a longer lifetime of a network. In the proposed work, the selection is based on the number of alive nodes subsists on the distance, energy, and delay.

Rest of the paper is organized as follows. In Section 2, relevant literature is briefly reviewed. In Section 3, the base station positioning model is presented with required mathematical models. The performance and evaluation of the proposed work are shown in Section 4. In Section 5 the concluding remarks are discussed.

\section{Related Works}

In 2014, Arkin et al. [1] focused on shallow tree topologies to maximize the network lifetime for energy efficiency on wireless sensor network optimization problem. However, Chanak et al. [3] identified an issue in WSN for analyzing the network due to improvisational nature. To overcome this problem in WSN they have proposed Mobile sink based distributed algorithm. Moreover, Snigdh et al. [7] worked on WSN, and they have proposed a method to choose the correct site for sink placement by means of placing the sink at optimal position. Likewise, Yang [36] worked on the positioning of multiple sink nodes by the simplified routing algorithm. On the Other hand, Mehra et al. [2] had worked on a cluster head selection based on localized parameters, and they have developed heterogeneity based clustering technique. Besides, Lanza-Gutierrez et al. [35] has developed two multi-objective evolutionary methods such as SPEA-2 (Strength Pareto Evolutionary Algorithm) and NSGA-II (Genetic Algorithm for multiple objectives). Wherever, Salman [37] had worked on wireless sensor networks (WSNs) for the localization of sensor nodes to promote numerous new applications. Furthermore, Medhane and Sangaiah [38] worked on (PMS) Position Monitoring System it is a wireless networking application to track and monitoring the objects to position in the wireless network.

\section{Problem Statement:}

Numerous research contributions have been made to address the problem of the base station positioning system. Their review outcomes are summarized in Table 1. The LEACH method used in [1] has advantages such as scalability and increased network lifetime. Nevertheless, it does not have inter-cluster communication. The greedy algorithm [3] has been experimentally demonstrated for its simplicity and efficiency. However, the viability greedy algorithm is limited based on the problem complexity. On the other hand, the dynamic source routing [7] is known for its reduction in control overhead, while it has drawbacks such as delay and performance degradation. The linear programming [2] can improve the decision quality with high flexibility. However, it has disadvantages such as determining an unrealistic location for the base station. The Evolutionary algorithm [35] is found to be efficient, but it remains expensive. The Greedy algorithm used in [36] has advantages like good convergence and it is appropriate for the flexible distribution network. However, it suffers from poor modelling of the fitness function and its limitation to real-time performance. The weighted least squares (WLS) algorithm [37] has benefits such as interoperability, efficiency and precise. However, the precision highly relies on selecting appropriate weights.

The dynamic programming [38] can determine the sub-optimal solution, but it does not meet the principle of optimality. The advantages and significant challenges present in recent works are shown in Table 1. Moreover, genetic algorithm (GA) [36] is found to be promising for base station positioning, despite evolutionary algorithm [35] and greedy algorithm [3] are reported in the literature. The greedy algorithm [3] is mainly adopting the comparative study between two different potential locations and defining the optimal location for base station positioning, whereas the evolutionary algorithm [35] is older than GA [36].

\section{Base station positioning for WSN}

\subsection{Base station positioning model}

WSN consists of $\mathrm{N}$ sensor nodes with one base station $\mathrm{B}_{\mathrm{S}}$ as shown in Fig. 1. Moreover, sensor nodes are grouped into several clusters $n_{a}$, and each cluster has one cluster head $\mathrm{CH}$. Here, wireless links between $\mathrm{N}$ sensor nodes indicate the direct communication within a radio range. The sensor nodes exhibit a random distribution in the network area of dimension $Y_{i}$ and $Z_{i}$. Generally, the Base station $\mathrm{B}_{\mathrm{S}}$ is positioned in the central location, i.e., $\left\{0.5 \mathrm{Y}_{\mathrm{i}}, 0.5 \mathrm{Z}_{\mathrm{i}}\right\}$ to collect the data from the nodes and the coordinate values of $\mathrm{Y}_{\mathrm{p}}$ and $\mathrm{Z}_{\mathrm{p}}$ are used to measure the position of sensor nodes. Since the practical network has randomly distributed nodes, the location of the base station cannot be defined. In addition, a cluster head $\mathrm{CH}$ routing method is used to 
transfer the data from each sensor node to the base station.

In WSN, the $\mathrm{B}_{\mathrm{S}}$ positioning is a major factor to maximize the lifetime of sensor nodes. The network lifetime is described as the time until the sensor node $\mathrm{N}$ uses up its energy. Therefore, to achieve high network lifetime, the data created by each sensor node are permitted to be routed to the $\mathrm{B}_{\mathrm{S}}$ via a multipath. Here, the main aim is to reduce the distance between the cluster head and the base station to minimize the overall energy consumptions. Hence, self-adaptive PSO algorithm is developed to positioning the base station, and it is used to decrease the distance between sensor nodes and the base station.

\subsection{Objective model}

The main aim of SAPSO algorithm is to determine the position of the base station. The appropriate selection of base station is modelled using the fitness function. The fitness function is derived based on the distance between the cluster head and the base station. Therefore, the main aim of our proposed self-adaptive algorithm is used to minimize the distance the between the base station and cluster head. The adopted objective model is given in Eq. (1), where, $n$ refers to number of nodes in a cluster, $d$ refers the distance between cluster head and the base station and c refers to total number of cluster, $f_{i}$ refers the fitness function. The area of the network is considered here as $100 \mathrm{~m} \times 100 \mathrm{~m}$.

$$
f_{i}=\frac{1}{\sum_{i=1}^{c} \frac{n \times d}{\sqrt{\text { Area }}}}
$$

The objective model is solved using SAPSO, which is substantially different from PSO in determining the inertia weight. In PSO, the inertia weight is said to be constant, whereas SAPSO determines the weight based on the improvement exhibited by the particles in terms of fitness function. GA [36] is already known for its genetic operators based updating, which is highly different from the updating process of SAPSO, which performs velocity based updating to determine the optimal position for the base station. The updating process leads faster convergence than conventional algorithms.

Table 1.Review on the state-of-the-art methodologies in Base station positioning

\begin{tabular}{|c|c|c|c|}
\hline $\begin{array}{c}\text { Author } \\
\text { [citation] }\end{array}$ & $\begin{array}{c}\text { Adopted } \\
\text { methodology }\end{array}$ & Features & Challenges \\
\hline Mehra et al. [2] & LEACH & $\begin{array}{l}\text { Provides scalability to network } \\
\text { Single-hop routing is possible from sensor node } \\
\text { It increases the lifetime of network }\end{array}$ & $\begin{array}{l}\text { There is no inter-cluster communication } \\
\text { Cluster Head }(\mathrm{CH}) \text { are not uniformly } \\
\text { distributed }\end{array}$ \\
\hline Arkin et al. [1] & $\begin{array}{l}\text { Linear } \\
\text { programming } \\
\text { algorithm }\end{array}$ & $\begin{array}{l}\text { It is suitable for solving complex problems. } \\
\text { Helps in simplicity } \\
\text { It improves in quality of decision } \\
\text { More flexible }\end{array}$ & $\begin{array}{l}\text { Limit on the possible solution. } \\
\text { Unrealistic outcome. }\end{array}$ \\
\hline $\begin{array}{l}\text { Chanak et al. } \\
\text { [3] }\end{array}$ & Greedy algorithm & $\begin{array}{l}\text { Simplicity } \\
\text { Efficiency } \\
\text { Cost is driven by exhaustive search }\end{array}$ & $\begin{array}{l}\text { Greedy is not automatic } \\
\text { Not suited for all problem models. }\end{array}$ \\
\hline $\begin{array}{l}\text { Snigdh et al. } \\
\text { [7] }\end{array}$ & $\begin{array}{l}\text { Dynamic Source } \\
\text { Routing }\end{array}$ & $\begin{array}{l}\text { Table driven approach is eliminated } \\
\text { Efficiently reduces the control overhead }\end{array}$ & $\begin{array}{l}\text { Performance degrades rapidly with } \\
\text { increasing mobility } \\
\text { Delay is higher than table-driven protocols }\end{array}$ \\
\hline $\begin{array}{l}\text { Lanza- } \\
\text { Gutierrez et al. } \\
{[35]}\end{array}$ & $\begin{array}{l}\text { Evolutionary } \\
\text { algorithm }\end{array}$ & $\begin{array}{l}\text { Simpler channel equalization. } \\
\text { Spectrum efficiency is very high. }\end{array}$ & $\begin{array}{l}\text { Problem injoints semi-blind CE and turbo } \\
\text { MUD in communication systems. } \\
\text { To done optimal solution for CE } \\
\text { optimization. It is very expensive }\end{array}$ \\
\hline Yang [36] & Genetic algorithm & $\begin{array}{l}\text { Operation speed } \\
\text { Good convergence } \\
\text { Suitable for flexible distribution network }\end{array}$ & $\begin{array}{l}\text { Poorly known fitness function affects } \\
\text { performance } \\
\text { Limited real-time performance }\end{array}$ \\
\hline Salman [37] & $\begin{array}{l}\text { weighted least } \\
\text { squares (WLS) } \\
\text { algorithm }\end{array}$ & $\begin{array}{l}\text { Efficient method } \\
\text { Easily interpretable } \\
\text { Most precise parameter }\end{array}$ & $\begin{array}{l}\text { Assumption on weights are known exactly } \\
\text { Difficult to assess } \\
\text { Results are unpredictably affected } \\
\end{array}$ \\
\hline $\begin{array}{l}\text { Medhane and } \\
\text { Sangaiah [38] }\end{array}$ & $\begin{array}{l}\text { Dynamic } \\
\text { programming }\end{array}$ & $\begin{array}{l}\text { Enables to develop the sub solution for a large } \\
\text { program } \\
\text { The sub solution is easier to maintain and } \\
\text { debug. }\end{array}$ & $\begin{array}{l}\text { The dynamic programming does not satisfy } \\
\text { the principle of optimality problems. } \\
\text { Difficulty in tracking the number of partial } \\
\text { solutions. }\end{array}$ \\
\hline
\end{tabular}




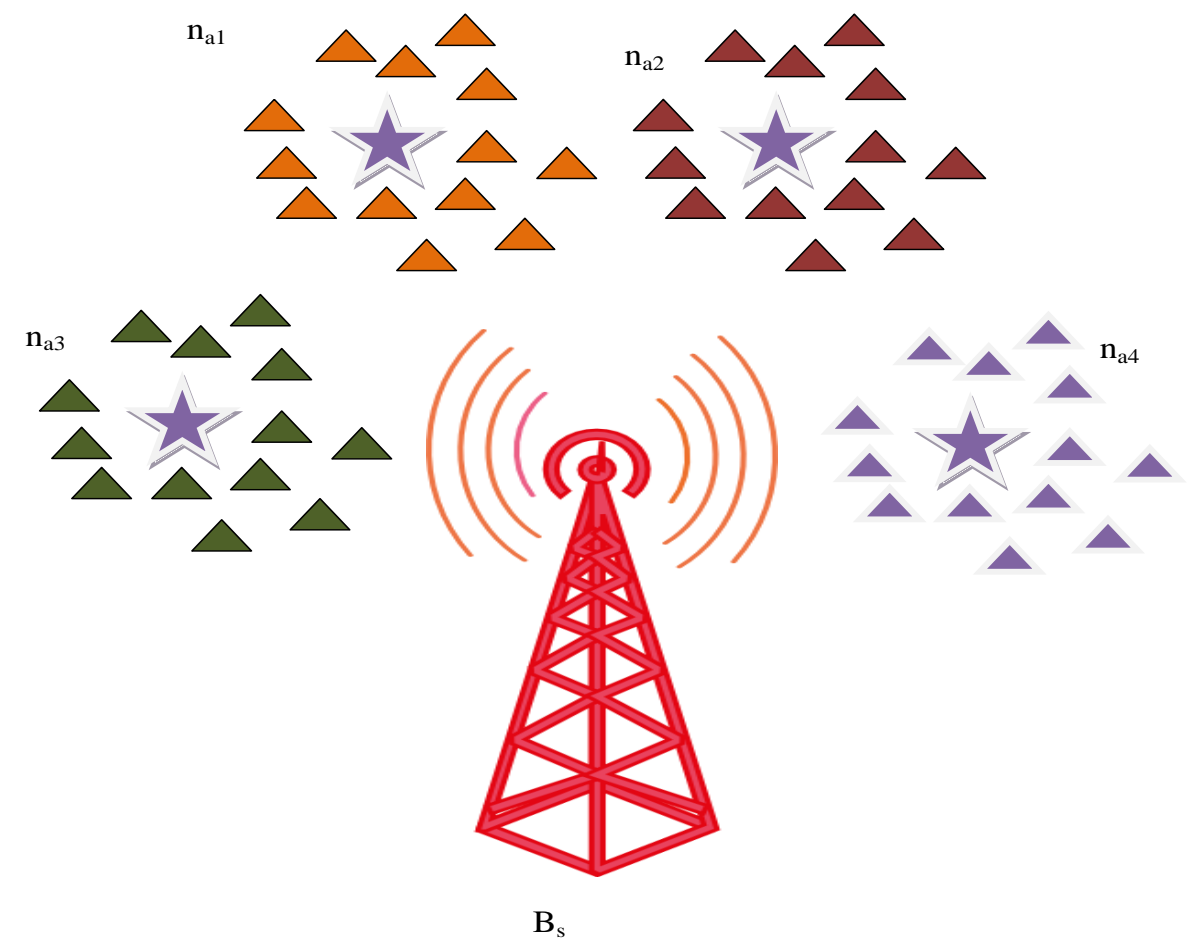

Figure.1Typical wireless sensor network with sensor nodes, grouped into 4 clusters.

\subsection{Conventional PSO and GA [36]}

In 1995, Kennedy and Eberhart developed a random optimization technique by the population called as Particle Swarm Optimization (PSO). They have shown the non-linear optimization method based on the bird's flock behavior. Fig. 2 describes the flow chart of conventional PSO.

The PSO algorithm follows three global variables

1. Target value

2. A Global best $\left(\mathrm{O}_{\mathrm{g}}\right)$ value, representing which particle data is presently nearest to the target.

3. Halt value: If the target value is not found then the halt value denotes when to stop the algorithm.

In PSO algorithm each particle consists of

1. Data representation, indicating the potential result.

2. The velocity value, representing the extent to which the data is altered.

3. $\left(\mathrm{O}_{\mathrm{f}}\right)$ Personal best value, representing the nearest particle data have ever come to the target.

In genetic algorithm, based on the fitness function each individual is estimated and combined with others at each iteration. Here, the expected number of times an individual is selected for recombination is proportional to its fitness relative to the rest of the population. Moreover, the crossover and mutation operation helps to create the new individuals. a) Crossover: The Crossover operation is performed by selecting a random location in the genetic string of the parents.

b) Mutation: The mutation operation for occasional disturbances in the crossover operation by inverting one or more genetic elements during reproduction.

\author{
Algorithm: Pseudo code for Conventional GA \\ Begin GA \\ $\mathrm{c}=0$ generation counter \\ Initialize the population \\ Evaluate the population G(c) \\ While not done do \\ $\mathrm{c}=\mathrm{c}+1$ \\ Select G(c) from G(c-1) \\ Crossover G(c) \\ Mutate G(c) \\ Evaluate G(c) \\ End while \\ End GA
}

\subsection{Proposed algorithm}

In this paper, proposed self-adaptive particle swarm optimization (SAPSO) algorithm is used to balance the system with inertia weight. The adaptive inertia weight scheme is provided to establish the position of a particle for each iteration.

In proposed method, the multi-dimensional search space is initialized for a random swarm of $n$ 
particles. Each particle exhibits a point in solution and relative fitness function in the randomized swarm to obtain the optimal solution. For example, let a swarm consists $\mathrm{n}$ particles, and it moves around $d$ dimensional search space. Suppose that, the velocity and the position of the $\mathrm{p}^{\text {th }}$ particle at the $\mathrm{i}^{\text {th }}$ iteration

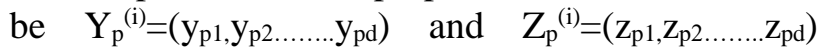
respectively. Here, $\mathrm{O}_{\mathrm{p}}{ }^{(\mathrm{i})}=\left(\mathrm{O}_{\mathrm{p} 1,} \mathrm{O}_{\left.\mathrm{p} 2 \ldots \ldots . . \mathrm{O}_{\mathrm{pd}}\right)}\right)$ and

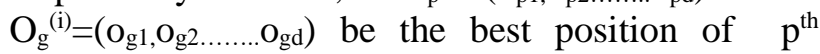
particle (i.e.) attained so far by itself is known as $\mathrm{O}_{\mathrm{f}}$ and best position obtained so far by the whole population is called as $\mathrm{O}_{\mathrm{g}}$. The velocity and the position of the $\mathrm{p}^{\text {th }}$ particle for next iteration can be determined as

$$
\begin{gathered}
Z_{i d}^{(i+1)}=x_{p}^{(i+1)} \times z_{p d}^{(i)}+t_{1} \times f_{1}^{(i+1)} \times\left(O_{p d}^{(i)}-Y_{p d}^{(i)}\right)+t_{2} \times f_{2}^{(i+1)} \times\left(O_{g d}^{(i)}-Y_{i d}^{(i)}\right) \\
Y_{p d}^{(i+1)}=Y_{i d}^{(i)}+Z_{p d}^{(i+1)}
\end{gathered}
$$

Here, $t_{1}$ and $t_{2}$ denotes the two positive constants known as social acceleration constant coefficient and the cognitive acceleration constant bcoefficient. Moreover, $\mathrm{f}_{1}{ }^{(\mathrm{i}+1)}$ and $\mathrm{f}_{2}{ }^{(\mathrm{i}+1)}$ indicates the random function within a $[0,1]$ range. Subsequently, $x_{p}{ }^{(i+1)}$ indicates the inertia factor of $\mathrm{p}^{\text {th }}$ particle at $(i+1)^{\text {th }}$ iteration. Each successive iteration the inertia weight is updated until it reaches the best solution.

\section{Pseudo code for Proposed Algorithm}

Input: $t_{1}$ and $t_{2}$

Initialization

For each $p^{\text {th }}$ particle in population

For number of particles

Initialize

$$
X_{p}^{(i)} \text { and } Z_{p}^{(i)}
$$

Calculate $r$

Initialize

$$
O_{p}^{(i)} \text { and } O_{g}^{(i)} ; \forall_{p} \leq n
$$

end

$$
x_{p}^{(i)}=0
$$

While

(termination condition, $\mathrm{i}$ is false)

$$
i=0
$$

For

$p=0 ;$ number of particles

For $d=1$; number of dimensions

$$
Z_{i d}^{(i+1)}=x_{p}^{(i+1)} \times z_{p d}^{(i)}+t_{1} \times f_{1}^{(i+1)} \times\left(O_{p d}^{(i)}-Y_{p d}^{(i)}\right)+t_{2} \times f_{2}^{(i+1)} \times\left(O_{g d}^{(i)}-Y_{i d}^{(i)}\right)
$$

check the velocity boundaries

$$
Y_{p d}^{(i+1)}=Y_{i d}^{(i)}+Z_{p d}^{(i+1)}
$$

end

If

$$
\begin{aligned}
& \left(r\left(Y_{p}^{i+1}\right)<r\left(O_{p}^{(i)}\right)\right) \\
& i=i+1 \\
& O_{p}=Y_{p}^{(i+1)} \\
& \text { If } \quad\left(r\left(Y_{p}^{(i+1)}\right)<r\left(O_{g}^{(i)}\right)\right) \\
& \qquad O_{g}=Y_{p}^{(i+1)}
\end{aligned}
$$

If

End

End

Update $\partial_{p}$ using Eq. (5).

Update $x_{p}$ using Eq. (4).

End

The self-adaptive inertia weight is deliberate by the following equation

$$
x_{p}^{(i+1)}=x_{p}^{(i)}+\left|f_{p}^{i+1} \times\left(x_{p}^{(i)}-x_{p}^{(i-1)}\right) \times \partial_{p}^{(i+1)}\right|
$$

Here, $\mathrm{f}_{\mathrm{p}}^{(\mathrm{i}+1)}$ represents the uniformly distributed random variables within a range $[0,1]$ at iteration $(\mathrm{i}+1)^{\mathrm{th}}, \mathrm{r}\left(\mathrm{Y}_{\mathrm{p}}^{(\mathrm{i}-1)}\right)$ and $\mathrm{r}\left(\mathrm{Y}_{\mathrm{p}}^{(\mathrm{i})}\right)$ revealed the fitness function of a particle $\mathrm{p}^{\text {th }}$ in $\mathrm{i}^{\text {th }}$ and (i-1) $)^{\text {th }}$ iterations respectively. Moreover, $\partial_{\mathrm{p}}{ }^{i+1}$ denotes the selfadaptive ratio of $(\mathrm{i}+1)^{\text {th }}$ iteration as illustrated below

$$
\partial_{p}^{(i+1)}=\frac{r\left(Y_{p}^{(i-1)}\right)-r\left(Y_{p}^{(i)}\right)}{r\left(Y_{p}^{(i-1)}\right)}
$$

\section{Results and Discussion}

\subsection{Simulation Setup}

In this section, the performance of the proposed self-adaptive approach is investigated through extensive simulation in MATLAB R2015a. The performance study is phased out by analyzing the energy efficiency with optimal positioning and without positioning. When studying the need of base station positioning, we compare the performance of SAPSO with traditional PSO in determining the optimal position for the base station.

Here, the proposed SAPSO is compared along with the Artificial Bee Colony-Dynamic Scout bee (ABC-DS) algorithm, Particle Swarm Optimization (PSO) and Firefly with Dual Update Process (FFDUP) algorithm. Moreover, the comparison of the proposed method in the simulation results is referred from [36]. Table 2 shows the details of experimental parameters considered during network setup. Similarly, Table 3 illustrates the parameters used in the algorithm. 


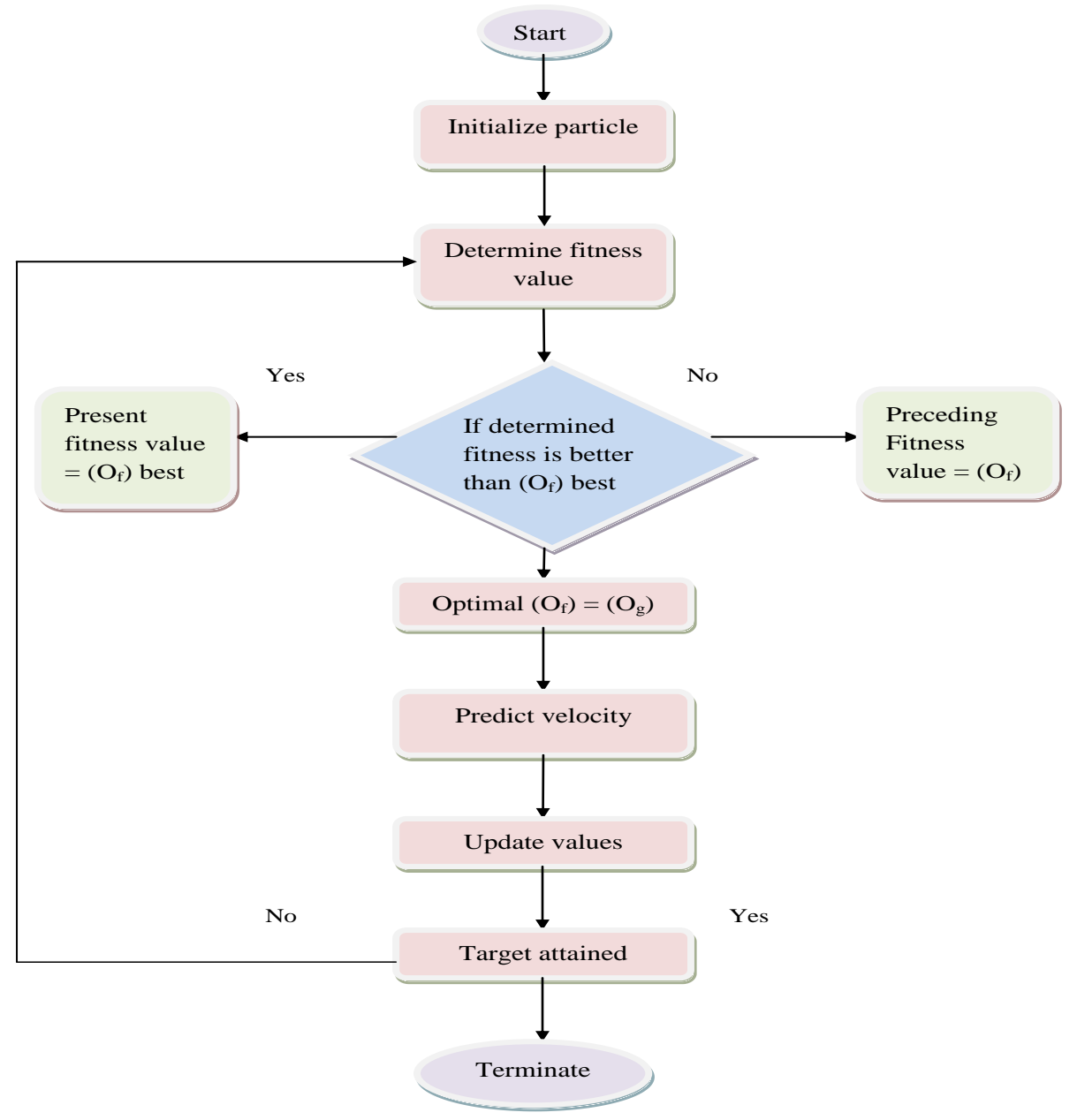

Figure.2 Flow chart of conventional PSO

Table 2. List of Parameters

\begin{tabular}{|l|l|}
\hline Parameter & Value \\
\hline Network size & $100 \mathrm{~m} \times 100 \mathrm{~m}$ \\
\hline Node number & 100 \\
\hline Node distribution & Random distributed \\
\hline Initial energy node & $0.5 \mathrm{~J}$ \\
\hline
\end{tabular}

Table 3. List of Parameters used in algorithm

\begin{tabular}{|l|l|}
\hline Parameter & Description \\
\hline $\mathrm{P}$ & Particle \\
\hline $\mathrm{t}_{1}, \mathrm{t}_{2}$ & Positive constants \\
\hline $\mathrm{f}_{1}, \mathrm{f}_{2}$ & Random function \\
\hline $\mathrm{X}_{\mathrm{p}}$ & Inertia factor of $\mathrm{p}^{\text {th }}$ particle \\
\hline $\mathrm{r}$ & Fitness function \\
\hline $\mathrm{Y}_{\mathrm{p}}$ & Velocity of particles \\
\hline $\mathrm{Z}_{\mathrm{p}}$ & Position of particles \\
\hline $\mathrm{O}_{\mathrm{f}}$ & Best position of $\mathrm{p}^{\text {th }}$ particle \\
\hline $\mathrm{O}_{\mathrm{g}}$ & Best position of the whole population \\
\hline$\partial_{\mathrm{p}}$ & Self-adaptive ratio \\
\hline
\end{tabular}

Table 4. Statistical analysis on the basis of cost function achieved by GA, PSO and SAPSO

\begin{tabular}{|l|l|l|c|}
\hline STATISTICS & GA & PSO & SAPSO \\
\hline Best & 3.7003 & 3.7003 & 3.7003 \\
\hline Worst & 3.7129 & 3.706 & 3.706 \\
\hline Mean & 3.7027 & 3.702 & 3.7009 \\
\hline Median & 3.7003 & 3.7003 & 3.7003 \\
\hline Standard Deviation & 0.00429 & 0.002774 & 0.001816 \\
\hline
\end{tabular}

International Journal of Intelligent Engineering and Systems, Vol.10, No.2, 2017

DOI: $10.22266 /$ ijies2017.0430.19

\subsection{Convergence analysis}

In this section, the convergence analysis of the existing and the proposed algorithm is performed using the fitness function. Fig. 3 depicts the function of both the existing PSO, GA and proposed SAPSO algorithm. The cost function of the existing GA algorithm remains constant in 3.73 from 0 to 85 iterations. Similarly, the PSO algorithm also remains constant in 3.72 from 0 to 80 iteration. But, the proposed SAPSO gradually decrease at 3.75 from 0 to 60 iterations and remains constant up to 100th iteration. Fig. 3 represents that the cost function of proposed SAPSO is $21 \%$ higher than of the existing PSO. Similarly, the cost function of the proposed SAPSO is $80 \%$ higher than the existing GA method. Hence, the performance of the proposed algorithm is superior to the conventional PSO algorithm.

Table 4 demonstrates that the statistical analysis for both the existing PSO and the proposed SAPSO method by the cost function. Here, the proposed algorithm shows better results than the conventional convergence graph, which is plotted based on the cost 
algorithm in terms of statistical parameters such as best as 3.7003, worst as 3.706, and mean as 3.7009, and median as 3.7003 and standard deviation as 0.001816 .

\subsection{Base station positioning on CHS framework}

Fig. 4 demonstrates the positioning of a base station regarding the proposed and the existing system. In Fig. 4 (a) depicts the number of alive nodes subsists on the distance, energy and delay until the completion of a number of rounds. It is observed that the number of the alive nodes for the Propsed SAPSO is $90 \%$ higher than the existing GA algorithms when simulated with 2000 rounds. Fig. 4 (b) shows that the number of alive nodes remains same for both the proposed and existing algorithms, but at round 2000 the proposed algorithm gets slightly increases than the existing algorithm regarding security. Fig. 4 (c) depicts the 37 number of alive nodes are subsisted while considering security, distance, energy, and delay until round 2000 for the SAPSO algorithm. Moreover, the Fig. 4 (c) shows that the proposed SAPSO is $80 \%$ better in terms of number of alive nodes than the conventional genetic algorithm. Hence, Fig. 4 shows that the proposed algorithm performs better than the existing ABC-DS, GA, FF-DUP and PSO algorithm.

Fig. 5 depicts the normalized network energy for 100 nodes. In Fig. 5 (a) the proposed SAPSO algorithm and the existing ABC- DS,GA and PSO algorithm remains same until the round 600 and from round 600 the proposed SAPSO algorithm slightly varies and then from round 800 the proposed algorithm gradually increase. Here, the proposed algorithm performs $28 \%$ higher than the existing PSO algorithm which shows that the proposed algorithm has more normalized energy than the existing algorithm. Fig. 5 (b) shows the normalized network energy regarding security. Here, till round 700 the proposed and the existing algorithm remains same and at round 700 both the proposed SAPSO algorithm and the existing PSO algorithm correlate with each other until round 1800 after that the proposed algorithm slightly increases. Moreover, the proposed algorithm performs $80 \%$ better in terms of normalized energy while comparing with the existing FF-DUP algorithm. The Fig. 5 (c) depicts that the proposed algorithm performs $10 \%$ better than the PSO algorithm. Furthermore, Fig. 5 (c) demonstrates that the proposed algorithm performs slightly better at round 2000 than the existing algorithm on the security, distance, energy and delay security, distance, energy, and delay.
Eventually, it can be asserted that the proposed base station positioning algorithm lays cornerstone to estimate the optimal position for the base stations not only based on the geographical distribution of the network, but also based on the communication link establishments, frequent communication links, characteristics of the routing protocols and many other features of the network. Moreover, the proposed algorithm is highly reliable for nonlinear objective model and so the base station positioning with diverse network characteristics can be highly successful compared with the conventional optimization algorithms.

\section{Conclusion}

This paper deals with the base station positioning, a self-adaptive particle swarm optimization (SAPSO) technique is proposed for WSN using fitness function. To prolong the lifetime of a sensor the distance between cluster head and the base station is minimized. The performance of the proposed method is compared with the other conventional methods such as Artificial Bee Colony-Dynamic Scout bee (ABC-DS) algorithm, Particle swarm optimization (PSO), Genetic algorithm (GA) and Firefly with Dual Update Process (FFDUP) algorithm in terms of energy, delay, and distance. Besides, to show the improvement of proposed method with the existing method, we have calculated the number of alive nodes and the network energy on the basis of optimized positioning of the base station. It can be observed that the SAPSO outperforms better than the existing algorithm. Finally, the simulation result demonstrates that the proposed algorithm is used to reduce the distance of the base station from the cluster head in a significant manner.

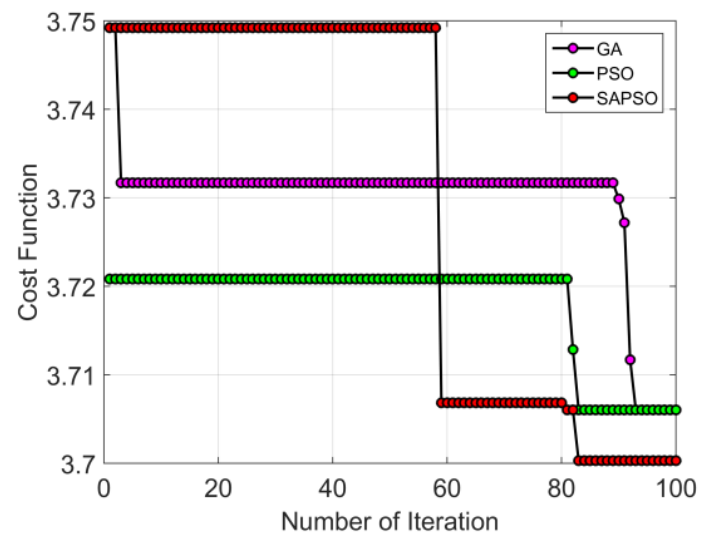

Figure.3 Analysis of the convergence graph 


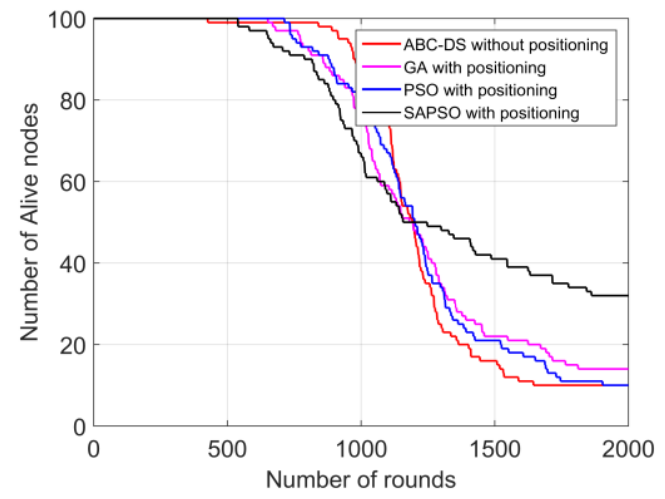

(a)

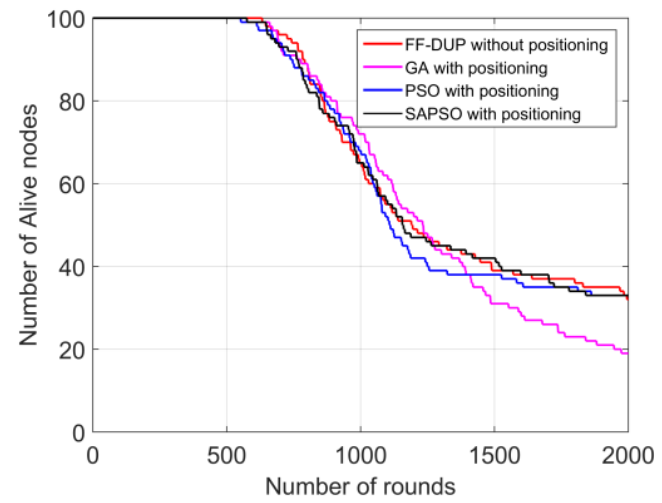

(b)

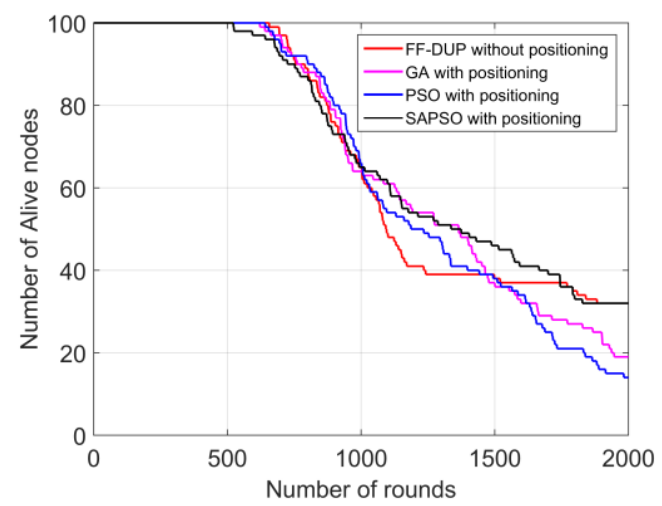

(c)

Figure.4.Graphical representation of Base station positioning on CHS framework (a) alive nodes on distance, energy, and delay (b) alive nodes on security (c) alive nodes on security, distance, energy, and delay.

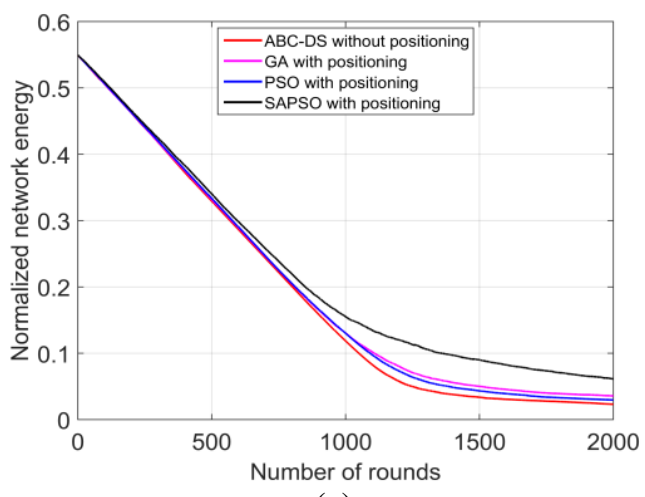

(a)

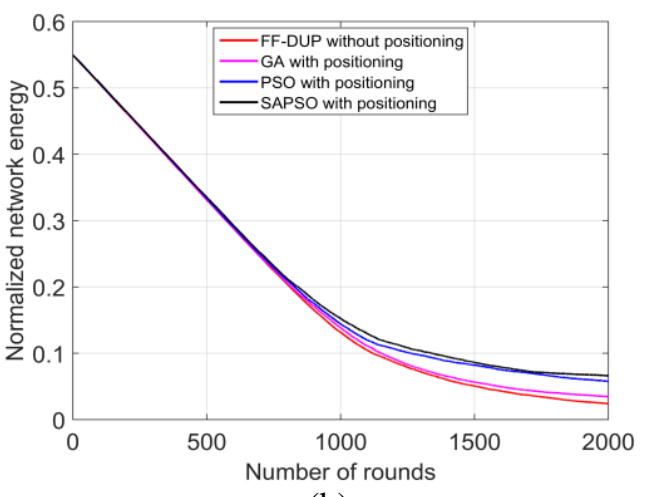

(b)

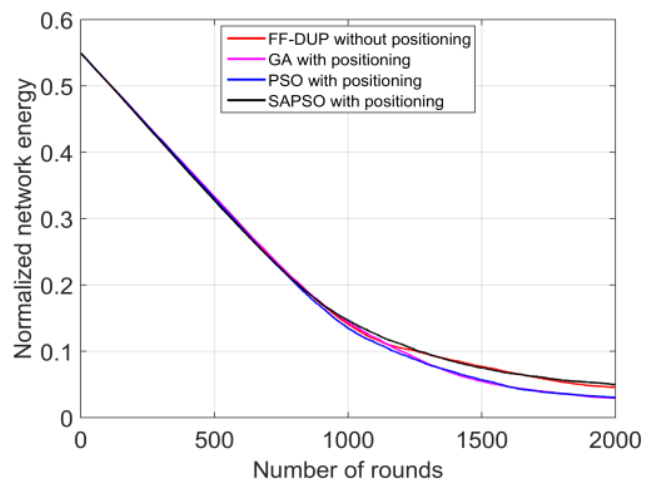

(c)

Figure .5Normalized network energy on distance, energy, and delay (b) Normalized energy on security (c) Normalized energy on security, distance, energy, and delay. 
Despite the fact that the base station positioning is a classical technique, less number of algorithms has been deployed for solving the problem. Moreover, the adopted algorithms are classical evolutionary algorithms such as genetic algorithm. Hence, we additionally included the genetic algorithm for comparative study along with the base algorithm for the proposed algorithm, i.e. PSO. As the advanced optimization algorithms are able to solve the problem, we have intended to carry out an extensive study over the performance of various advanced optimization algorithms in the near future.

\section{References}

[1] E.M. Arkina, A. Efratb, J.S.B. Mitchella, V.Polishchukc, S.Ramasubramani, S.Sankararamanb, J.Taherib, "Data transmission and base-station placement for optimizing the lifetime of wireless sensor networks," Ad Hoc Networks, Vol. 12, pp. 201-218, 2014.

[2] P.S.Mehra, M. N. Doja, B. Alam," Enhanced Stable Period for Two Level and Multilevel Heterogeneous Model for Distant Base Station in Wireless Sensor Network," Proceedings of the Second International Conference on Computer and Communication Technologies, Vol. 379 ,pp 751-759, 2015.

[3] P.Chanaka, I.Banerjeea, R. Simon Sherrattb,"Mobile sink based fault diagnosis scheme for wireless sensor networks," Journal of Systems and Software, Vol. 119, pp. 45-57, 2016.

[4] K. Akkaya, M. Younis and W. Youssef, "Positioning of Base Stations in Wireless Sensor Networks," IEEE Communications Magazine, Vol. 45, No. 4, pp. 96-102, 2007.

[5] L. Chen, W. Wang, H. Huang and S. Lin, "On Time-Constrained Data Harvesting in Wireless Sensor Networks: Approximation Algorithm Design," IEEE/ACM Transactions on Networking, Vol. 24, No. 5, pp. 3123-3135, 2016.

[6] F. Yuan, Y. Zhan and Y. Wang, "Data Density Correlation Degree Clustering Method for Data Aggregation in WSN," IEEE Sensors Journal, Vol. 14, No. 4, pp. 1089-1098, 2014.

[7] I. Snigdha, D. Gosaina, N. Guptab, "Optimal sink placement in backbone assisted wireless sensor networks ," Egyptian Informatics Journal, Vol. 17, pp. 217-225, 2016.

[8] P. Gope and T. Hwang, "A Realistic Lightweight Anonymous Authentication Protocol for Securing Real-Time Application Data Access in Wireless Sensor Networks," IEEE Transactions on Industrial Electronics, Vol. 63, No. 11, pp. 7124-7132, 2016.
[9] P . Abouzar, D. G. Michelson and M. Hamdi, "RSSI-Based Distributed Self-Localization for Wireless Sensor Networks Used in Precision Agriculture," IEEE Transactions on Wireless Communications, Vol. 15, No. 10, pp.6638-6650, 2016.

[10] Y. Yu, "Consensus-Based Distributed Mixture Kalman Filter for Maneuvering Target Tracking in Wireless Sensor Networks," IEEE Transactions on Vehicular Technology, Vol. 65, No. 10, pp. 8669-8681, 2016.

[11] M. Ye, Y. Wang, C. Dai and X. Wang, "A Hybrid Genetic Algorithm for the Minimum Exposure Path Problem of Wireless Sensor Networks Based on a Numerical Functional Extreme Model," IEEE Transactions on Vehicular Technology, Vol. 65, No. 10, pp. 86448657, 2016.

[12] D. Izadi, J. Abawajy and S. Ghanavati, "An Alternative Clustering Scheme in WSN," IEEE Sensors Journal, Vol. 15, No. 7, pp. 4148-4155, 2015.

[13] R. V. Kulkarni and G. K. Venayagamoorthy, "Particle Swarm Optimization in WirelessSensor Networks: A Brief Survey," IEEE Transactions on Systems, Man, and Cybernetics, Part C (Applications and Reviews), Vol. 41, No. 2, pp. 262-267, 2011.

[14] P.D.H.Zadeha, C.Schlegelb, M.H. MacGregorb, "Distributed optimal dynamic base station positioning in wireless sensor networks", Computer Networks, Vol. 56, pp. 34-49, 2012.

[15] D. Jia, H. Zhu, S. Zou and P. Hu, "Dynamic Cluster Head Selection Method for Wireless Sensor Network," IEEE Sensors Journal, Vol. 16, No. 8, pp. 2746-2754, 2016.

[16] H. Lin, L. Wang and R. Kong, "Energy Efficient Clustering Protocol for Large-Scale Sensor Networks," IEEE Sensors Journal, Vol. 15, No. 12, pp. 7150-7160, 2015.

[17] P. S. Bithas, A. S. Lioumpas and A. Alexiou, "Mitigating shadowing effects through clusterhead cooperation techniques," IET Networks, Vol. 2, No. 2, pp. 71-80, 2013.

[18] J. S. Leu, T. H. Chiang, M. C. Yu and K. W. $\mathrm{Su}$, "Energy Efficient Clustering Scheme for Prolonging the Lifetime of Wireless Sensor Network With Isolated Nodes," IEEE Communications Letters, Vol. 19, No. 2, pp. 259262, 2015.

[19] J. RejinaParvin and C. Vasanthanayaki, "Particle Swarm Optimization-Based Clustering by Preventing Residual Nodes in Wireless Sensor Networks," IEEE Sensors Journal, Vol. 15, No. 8, pp. 4264-4274, 2015. 
[20] H. Fotouhi, M. Alves, M. Z. Zamalloa and A. Koubâa, "Reliable and Fast Hand-Offs in LowPower Wireless Networks," IEEE Transactions on Mobile Computing, Vol. 13, No. 11, pp. 26202633, 2014.

[21] S.Tyagia, N. Kumarb,"A systematic review on clustering and routing techniques based upon LEACH protocol for wireless sensor networks," Journal of Network and Computer Applications, Vol. 36, pp. 623-645, 2013,

[22] S. H. Kang and T. Nguyen, "Distance Based Thresholds for Cluster Head Selection in Wireless Sensor Networks," IEEE Communications Letters, Vol. 16, No. 9, pp. 1396-1399, 2012.

[23] J. Wang, Y. Yin, J. Zhang, S. Lee and R. S. Sherratt, "Mobility based energy efficient and multi-sink algorithms for consumer home networks," IEEE Transactions on Consumer Electronics, Vol. 59, No. 1, pp. 77-84, 2013.

[24] A. Chelli, M. Bagaa, D. Djenouri, I. Balasingham and T. Taleb, "One-Step Approach for Two-Tiered Constrained Relay Node Placement in Wireless Sensor Networks," IEEE Wireless Communications Letters, Vol. 5, No. 4, pp. 448-451, 2016.

[25] A. Agarwal and K. Agarwal, "Performance evaluation of OFDM based WiMAX (IEEE 802.16d) system under diverse channel conditions," International Conference on Electrical, Electronics, Signals, Communication and Optimization (EESCO), pp. 1-5, 2015.

[26] L. Chen, W. Wang, H. Huang and S. Lin, "On Time-Constrained Data Harvesting in Wireless Sensor Networks: Approximation Algorithm Design," IEEE/ACM Transactions on Networking, Vol. 24, No. 5, pp. 3123-3135, 2016.

[27] M. Ye, Y. Wang, C. Dai and X. Wang, "A Hybrid Genetic Algorithm for the Minimum Exposure Path Problem of Wireless Sensor Networks Based on a Numerical Functional Extreme Model," IEEE Transactions on Vehicular Technology, Vol. 65, No. 10, pp. 86448657, 2016.

[28] T. Olasupo, C. E. Otero, I. Kostanic and S. Shaikh, "Effects of terrain variations in Wireless Sensor Network deployments," IEEE International $R F$ and Microwave Conference (RFM), Kuching, Malaysia, pp. 83-88, 2016.

[29] P.Chanaka, I.Banerjeea, R.S.Sherrattb, "Obstacle avoidance routing scheme through optimal sink movement for home monitoring and mobile robotic consumer devices," IEEE Transactions on Consumer Electronics, Vol. 60, No. 4, pp. 596-604, 2014.
[30] M.E. Migabo, K. Djouani, A.M. Kurien, T.O. Olwal,"Gradient-based Routing for Energy Consumption Balance in Multiple Sinks-based Wireless Sensor Networks" Procedia Computer Science, Vol. 63, pp 488-493, 2015.

[31] G. A. Shah, F. Alagoz, E. A. Fadel and O.B. Akan, "A Spectrum-Aware Clustering for Efficient Multimedia Routing in Cognitive Radio Sensor Networks," IEEE Transactions on Vehicular Technology, Vol. 63, No. 7, pp. 33693380, 2014.

[32] P. Nayak and A. Devulapalli, "A Fuzzy Logic-Based Clustering Algorithm for WSN to Extend the Network Lifetime," IEEE Sensors Journal, Vol. 16, No. 1, pp. 137-144, 2016.

[33] S.Ghafoor, M. H. Rehmanic, S.Choa, S-H. Parka,"An efficient trajectory design for mobile sink in a wireless sensor network ", Computers \& Electrical Engineering, Vol. 40, pp. 2089-2100, 2014.

[34] M. Ozger, E. Fadel and O. B. Akan, "Eventto-Sink Spectrum-Aware Clustering in Mobile Cognitive Radio Sensor Networks," IEEE Transactions on Mobile Computing, Vol. 15, No. 9, pp. 2221-2233, 2016.

[35] J.M. Lanza-Gutierrez, J.A. Gomez-Pulido, M.A. Vega-Rodríguez, J.M. Sanchez-Perez, "Relay Node Positioning in Wireless Sensor Networks by Means of Evolutionary Techniques", Autonomous and Intelligent Systems, Vol.7326, pp 18-25, 2012.

[36] S.H. Yang, "Optimization of Sink Node Positioning", Signals and Communication Technology, pp 129-141,2014.

[37] N. Salman, M. Ghogho and A. H. Kemp, "Optimized Low Complexity Sensor Node Positioning in Wireless Sensor Networks," IEEE Sensors Journal, Vol. 14, No. 1, pp. 39-46, 2014. [38] D.Vishwasrao and A.K. Sangaiah, "Source Node Position Confidentiality (SNPC) Conserving Position Monitoring System for Wireless Networks," International Journal of High Performance Systems Architecture, Vol. 6, No. 2, 2015. 